\title{
Metformin macht das Mikrobiom antidiabetisch
}

Fragestellungen: Wie beeinflusst Metformin die Darmmikrobiota von Patienten mit neu diagnostiziertem und noch unbehandeltem Diabetes, und welche Wechselwirkungen entstehen zwischen Metformin und den Darmbakterien?

Hintergrund: Vielen Menschen mit Typ-2-Diabetes (T2D) werden mit Metformin behandelt, aber dessen Wirkmechanismus ist nicht klar definiert. Studienergebnisse deuten darauf hin, dass die Darmmikrobiota der Wirkort des Medikaments ist.
Wu H, Esteve E, Tremaroli V et al. Metformin alters the gut microbiome of individuals with treatment-naive type 2 diabetes, contributing to the therapeutic effects of the drug. Nat Med. 2017 Jul;23(7):850-58.
Material und Methoden: In einer doppelblinden, randomisierten Studie erhielten 40 Patienten mit bis dahin noch unbehandeltem Diabetes für 4 Monate nur eine diätetische Therapie und Placebo oder Metformin.
Ergebnisse: Metformin zeigte im Vergleich zu Placebo starke Auswirkungen auf das Darm-Mikrobiom. Die Ergebnisse wurden in einer Teilmenge der Placebo-Gruppe verifiziert, die $6 \mathrm{Mo-}$ nate nach Studienbeginn auf Metformin umgestellt wurden. $\mathrm{Zu}$ dem führte die Übertragung der Mikrobiota von Spendern vor und vier Monate nach Behandlungsbeginn mit Metfomin auf keimfreie Mäuse zu einer Verbesserung der Glukosetoleranz bei den Mäusen, die Metformin-Mikrobiota erhielten.

Um die direkten Metformin-Mikrobiota-Wechselwirkungen genauer zu analysieren, wurde mithilfe eines sog. „Darmsimulators“ gezeigt, dass Metformin viele Signalwege aus zwei verschiedenen Bakterienstämmen und viele der durch Metformin regulierten Gene dieser Bakterienarten codieren kann, die für Metalloproteine oder -transporter zuständig sind.

Schlussfogerung: Diese Erkenntnisse unterstützen die Vorstellung, dass eine veränderte Darmmikrobiota - auch vermittelt durch Metformin - antidiabetische Wirkungen haben kann.

\section{- Kommentar von Dr. Marie-Christine Simon}

\section{Metallbindende Proteine sind wohl der Schlüssel}

Die Studie beschreibt, dass Metformin offenbar durch Veränderungen an den Bakterien im Darm antidiabetisch wirkt. Die Forscher konnten zeigen, dass es starke Auswirkungen auf die Zusammensetzung und Funktion des Mikrobioms hat, was zum therapeutischen Effekt beiträgt. Zudem zeigt diese Arbeit, dass Metformin mit Darmbakterien interagiert, möglicherweise durch die Regulierung der Metalloproteine, die bei vielen biologischen Prozessen entscheidend sind. Zu nennen sind Respiration, molekulare Reduktion von Sauerstoff, Oxidation von Wasser und Stickstofffixierung [1]. Interessanterweise sind einige Metallionen in diesen Proteinen, die wichtig sind für deren Funktion, Struktur und Stabilität, bekannt dafür, zur Pathophysiologie des T2D beizutragen [2]. Es ist seit vielen Jahren bekannt, dass Metformin an Metalle bindet [3]. Darüber hinaus zeigt eine aktuelle Studie, dass die Wirkungen von Metformin von seinen metallbindenden Eigenschaften abhängen [3]. Diese neue Arbeit von Wu et al. kann nun zeigen, dass es einen Zusammenhang zwischen Metformin und metallbindenden Proteinen gibt, die von der Darmmikrobiota produziert werden. Allerdings sind zusätzliche Studien nötig, um die mikrobiellen Metaboliten oder Proteine zu identifizieren und herauszufinden, wie sie den humanen Metabolismus verbessern und mit dem Wirtsmetabolismus interagieren.

Metformin ist bei übergewichtigen Typ-2-Diabetikern Mittel der Wahl. Wegen seiner niedrigen Kosten, dem hohen Sicherheitslevel und positiver Wirkungen auf Blutglukose und Herz-Kreislauf-Erkrankungen ist es die meistverschriebene Arznei bei T2D [4, 5]. Obwohl Metformin im Allgemeinen Hyperglykämien entgegenwirkt, indem die hepatische Gluko- neogenese gehemmt wird, und zwar durch Aktivierung der proteinkinase-(AMPK-)abhängigen und AMPK-unabhängigen Signalkaskaden der Leber, sammeln sich die Zeichen, dass sich weitere wesentliche Wirkmechanismen wie die verzögerte Glukoseresorption im Darm abspielen $[6,7,8,9,10,11,12,13]$.

\section{Literatur}

. Lu, Y. et al. Design of functional metalloproteins Nature. 2009

2. Fernández-Real, J.M. \& Manco, M. Lancet Diabetes Endocrinol. 2014

3. Logie, L. et al. Diabetes. 2012

4. Nathan, D.M. et al. Diabetes Care 2009

5. Pernicova, I. \& Korbonits, M. Nat. Rev. Endocrinol. 2014.

6. Zhou, G et al. J. Clin. Invest. 2001

7. Shaw, R.J. et al. Science 2005

8. Fullerton, M.D. et al. Nat. Med. 2013

9. Foretz, M. et al. Clin. Invest. 2010

10. Madiraju, A.K. et al. Nature 2014

11. Miller, R.A. et al. Nature 2013

12. McCreight, L.J., Bailey, C.J. \& Pearson, E.R. Diabetologia 2016.

13. Duca, F.A. et al. Nat. Med. 2015.

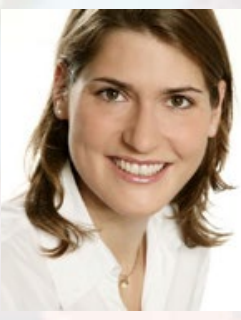

Marie-Christine Simon, PhD MSc RD

Bäckhed Lab, Wallenberg Laboratory,

University of Gothenburg

Bruna Stråket 16, 41345 Göteborg - Sweden Marie-Christine.Simon@wlab.gu.se 